\title{
Conocimiento de estudiantes primarios sobre el bosque y la biodiversidad de las Yungas Australes en la ciudad de San Salvador de Jujuy, Argentina
}

\author{
Alejandro Schaaf ${ }^{\varpi}$; Sofia Alcalde; Luis Rivera \& Natalia Politi \\ Instituto de Ecorregiones Andinas (INECOA), Universidad Nacional de Jujuy - Consejo Nacional de Investigaciones \\ Científicas y Técnicas (CONICET), San Salvador de Jujuy, Argentina. Fundación CEBio, San Salvador de Jujuy, Argentina
}

\begin{abstract}
Resumen. Las Yungas Australes poseen una biodiversidad sobresaliente y conforman uno de los ecosistemas más amenazados de la Región Andina. Históricamente, distintas actividades humanas les hicieron sufrir una presión no planificada. La educación en conservación puede ser una herramienta para valorar la biodiversidad. En este trabajo evaluamos el conocimiento de la biodiversidad de las Yungas por parte de estudiantes de séptimo grado de establecimientos educativos de la ciudad de San Salvador de Jujuy. Tomamos al azar cuatro establecimientos y encuestamos un total de 191 niños mediante un sistema de preguntas que abarcó dos ejes: a) la valoración de la función y la importancia de los bosques, y b) el reconocimiento de animales y plantas. El 88\% de los estudiantes encuestados considera que los bosques nativos son ecosistemas complejos que se desarrollan de forma natural y están compuestos por especies que por miles de años se adaptaron a las condiciones del lugar. El 44\% percibe que los bosques nativos son importantes por proveer bienes y servicios ecosistémicos, y por ser hábitat para un gran número de especies animales y vegetales. El $80 \%$ de los estudiantes considera que los parques nacionales constituyen la estrategia principal para conservar las especies. En proporción, las especies exóticas de árboles fueron adjudicadas más al bosque nativo que las nativas. Sólo los animales más carismáticos (e.g., el tucán y el yaguareté) fueron identificados de manera adecuada por más del 80\% de los estudiantes. Estos resultados sugieren la necesidad de realizar intervenciones que permitan difundir la importancia de los bosques nativos y mejorar el conocimiento sobre las especies nativas como estrategia para fomentar la conservación de la biodiversidad.
\end{abstract}

[Palabras clave: conservación, especies nativas, encuestas]

Aвstract. Knowledge by seventh grade students about forest and biodiversity of Southern Yungas in San Salvador de Jujuy city, Argentina. The Southern Yungas is one of the most endangered ecosystems of the Andean Region. Its outstanding biodiversity has suffered the unplanned pressure from different human activities. Conservation education could be a tool to value biodiversity. In this work, we assessed knowledge and perception of seventh grade students about Southern Yungas biodiversity in San Salvador de Jujuy city. Four schools were randomly selected and 191 students were surveyed through a questionnaire that allowed us to encompass two axes: perception and knowledge. Our results suggest that $88 \%$ consider native forests as complex ecosystems that naturally developed in an area and are composed by species that have adapted to local conditions over thousands of years, while $44 \%$ perceive that forest are important for providing goods, ecosystem services, and are habitat for many animals and plant species. Eighty percent of students consider that national parks constitute the main strategy for species conservation. Exotic tree species were proportionally more related to native forest than native tree species. Toucans and Jaguars were the animal native species most known by students $(>80 \%)$. Our results show that it is necessary to further work at schools to raise knowledge and perception among students of the importance of native forests and to improve the knowledge of Southern Yungas native species as a way to promote biodiversity conservation.

[Key words: conservation, native species, surveys]

Editora asociada: María Semmartin
Recibido: 11 de abril de 2018

Aceptado: 27 de septiembre de 2018 


\section{INTRODUCCIÓN}

El conocimiento que tienen las personas sobre la biodiversidad y su conservación se puede definir como el conjunto de comprensiones e interpretaciones que surgen del entorno que los rodea; en donde la forma en que conocen a los ecosistemas que los rodean puede afectar a la conservación de la biodiversidad (Pilgrim et al. 2008; Bickford et al. 2012). Este conocimiento adquirido depende de factores socio-demográficos como, por ejemplo, la vinculación con los recursos naturales, el estilo de vida, la filiación cultural o los accesos a medios de comunicación (Durand 2008; Pilgrim et al. 2008; Huddart-Kennedy et al. 2009; Campos et al. 2013; Trillo et al. 2016).

El conocimiento que adquieran las personas determinará sus actitudes con respecto a los bosques nativos, su funcionamiento y su biodiversidad (Pooley and O'Connor 2000). Es por esto que la educación en escuelas primarias juega un rol fundamental en el conocimiento y la valoración de los ecosistemas, su flora y fauna asociada a cada lugar. Dado que los niños poseen una gran curiosidad, las experiencias educativas con la naturaleza pueden despertar el deseo de aprender y de conocer cómo están compuestos los bosques o qué especies animales y vegetales se pueden encontrar allí (Sobel 1999; Furman and de Podestá 2010; Baranzelli et al. 2015). En particular, en la Argentina, estudios previos realizados por Campos et al. (2013) y Baranzelli et al. (2015) en escuelas primarias de Córdoba, Mendoza y San Juan documentaron un escaso conocimiento de la biodiversidad nativa, mientras que el conocimiento de las especies exóticas es mayor. Principalmente, detectaron una familiarización con mascotas, animales domésticos y plantas ornamentales. A su vez, también hallaron diferencias en las fuentes de información que usan (i.e., libros, internet $\mathrm{u}$ hogar), en las edades de los niños y niñas, y en el género y el lugar de residencia (urbano o rural). Esta falta de conocimiento sobre especies nativas, sumado a limitaciones en el conocimiento ecológico y ambiental, contribuyen a que los niños se alejen de la naturaleza, de la conservación y del entorno natural inmediato que los rodea (Jordan et al. 2009; Bickford et al. 2012). Por lo tanto, en las escuelas resulta indispensable fortalecer la educación ambiental, sobre todo en ciudades o en localidades cuyos ecosistemas están amenazados por actividades antrópicas (Sudarmadi et al. 2001; Campos et al. 2013).
Si bien existen estudios realizados en el centro del país, no hallamos trabajos similares en la región noroeste, donde actualmente la ecorregión delas Yungas Australes, en conjunto con la Selva Misionera, contienen más del $50 \%$ de la biodiversidad del país (Brown et al. 2009). Las Yungas Australes, además, poseen un número elevado de endemismos (e.g., rana marsupial [Gastrotheca gracilis], el mirlo de agua [Cinclus schulzi]), especies amenazadas a nivel nacional (como el yaguareté [Panthera onca]) e internacional (Guacamayo verde [Ara militaris]), a la vez que brindan a los humanos una gran cantidad de bienes (e.g., leña, frutos y carne de monte) y servicios ecosistémicos (e.g., la captación de agua) (Brown et al. 2005). Hoy, estos bosques están considerados como uno de los ecosistemas más amenazados debido a la alta tasa de transformación para uso agrícola y urbanización. Se estima que un $30 \%$ de la superficie original fue transformada, y los sectores remanentes están siendo degradados por ganadería extensiva y la extracción forestal no planificada (Brown et al. 2005).

En este trabajo evaluamos el conocimiento por parte de los estudiantes de séptimo grado sobre las Yungas Australes y su biodiversidad en escuelas primarias de la ciudad de San Salvador de Jujuy, Argentina. Teniendo en cuenta los trabajos previos realizados en el centro del país (Campos et al. 2013; Baranzelli et al. 2015), planteamos la hipótesis de que el conocimiento de las especies dependerá de su condición de exóticas o nativas y, a la vez, de si son o no carismáticas. Esperamos que los alumnos estén más familiarizados con las especies exóticas y las carismáticas.

\section{Materiales y Métodos}

El trabajo se llevó a cabo en cuatro instituciones educativas que se encuentran ubicadas dentro del ejido urbano de San Salvador de Jujuy, provincia de Jujuy, noroeste argentino. Los estudiantes de estas escuelas viven en la ciudad, que se encuentra ubicada en la ecorregión de las Yungas Australes. Dos escuelas son de gestión privada y dos de gestión pública, y sus estudiantes presentan características socioeconómicas similares. Estas escuelas concedieron las autorizaciones para llevar adelante este trabajo. Encuestamos un total de 191 estudiantes de séptimo grado (entre 12 y 13 años de edad). Para detectar los conocimientos de los estudiantes en los diferentes colegios, no se realizaron charlas sobre la temática antes o durante 
el desarrollo de las encuestas. Por lo tanto, este trabajo se basó en una encuesta sobre conocimientos previos. De esta forma se pudo detectar sus mayores falencias en cuanto a bosques, animales y plantas de las Yungas. El tiempo destinado a las encuestas fue de aproximadamente una hora por colegio.

La encuesta constó de cinco ítems; cuatro fueron de opción múltiple y uno de identificación de especies animales de las Yungas Australes (ver Anexo). Por un lado, consideramos preguntas generales sobre bosques para poder establecer la apreciación que los alumnos tienen sobre éstos. Luego, realizamos preguntas sobre plantas y animales. Para las plantas realizamos una lista con las exóticas y las nativas de las Yungas con la idea de determinar cuáles especies los estudiantes asocian a los bosques nativos. Respecto a los animales, se seleccionaron nueve especies nativas de las Yungas (e importantes desde el punto de vista de la conservación) para que los alumnos le coloquen a cada una su nombre común (ver Anexo). Para las preguntas con respuestas de opciones múltiples se determinó el porcentaje para cada una de las respuestas con respecto al total. Para la pregunta 4, los alumnos podían marcar varias o todas de las opciones de plantas listadas, por lo que especies nativas y las exóticas se podían adjudicar como propias de los bosques nativos. Mientras tanto, para la pregunta 5 se determinó la frecuencia en que cada especie animal fue correctamente identificada por los estudiantes. En esta última pregunta se consideraron correctas distintas sinonimias de los nombres comunes de los animales. Por ejemplo, fue aceptada como correcta la palabra 'loro', a pesar de que no se nombre específicamente a la especie (ver resultados).

\section{Resultados}

Del total de estudiantes encuestados en las cuatro escuelas $(n=191)$, para la pregunta 1 , $88 \%$ contestó la opción correcta ('Ecosistemas complejos que se desarrollan naturalmente y desde siempre en un área, y están compuestos por especies que por miles de años se han adaptado a las condiciones del lugar'). Para la pregunta 2,44\% de los alumnos respondió las opciones correctas ('Porque proporcionan beneficios y recursos tales como, suelo, agua,

Tabla 1. Preguntas realizadas a estudiantes de séptimo grado en cuatro escuelas primarias de la ciudad de San Salvador de Jujuy, Argentina, y porcentaje de estudiantes que marcaron cada una de las opciones ( $\mathrm{n}=191$ encuestas realizadas).

Table 1. Questions made to seventh grade students in four elementary schools in the city of San Salvador de Jujuy, Argentina, and percentage of students that marked each of the options ( $\mathrm{n}=191$ surveys conducted).

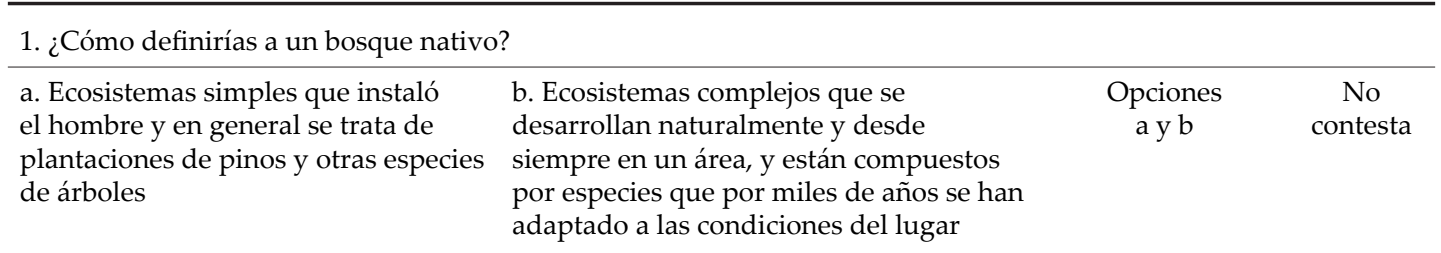

$11 \%$ $88 \%$

2. ¿Por qué son importantes los bosques?

\begin{tabular}{|c|c|c|c|c|c|c|c|}
\hline $\begin{array}{l}\text { a. Porque } \\
\text { proporcionan } \\
\text { beneficios y } \\
\text { recursos tales como, } \\
\text { suelo, agua, flora, } \\
\text { fauna, recreación } \\
\text { y paisaje para las } \\
\text { personas }\end{array}$ & $\begin{array}{l}\text { b. Porque se } \\
\text { puede extraer } \\
\text { madera y } \\
\text { otras plantas } \\
\text { de utilidad } \\
\text { para los seres } \\
\text { humanos }\end{array}$ & $\begin{array}{l}\text { c. Porque } \\
\text { son el lugar } \\
\text { donde viven } \\
\text { muchas } \\
\text { especies de } \\
\text { animales }\end{array}$ & $\begin{array}{c}\text { Opciones } \\
\text { a y b }\end{array}$ & $\begin{array}{c}\text { Opciones } \\
\text { a y c }\end{array}$ & $\begin{array}{c}\text { Opciones } \\
\text { b y c }\end{array}$ & $\begin{array}{l}\text { Opciones } \\
\mathrm{a}, \mathrm{b} \text { y c }\end{array}$ & $\begin{array}{c}\text { No } \\
\text { contesta }\end{array}$ \\
\hline $22 \%$ & $2 \%$ & $17 \%$ & $6 \%$ & $44 \%$ & $3 \%$ & $6 \%$ & $0 \%$ \\
\hline \multicolumn{8}{|c|}{ 3. ¿Cuál de estos lugares les parece el más importante para proteger a los animales y plantas nativas? } \\
\hline a. Zoológico & $\begin{array}{l}\text { b. Parque } \\
\text { nacional }\end{array}$ & c. Plaza & $\begin{array}{c}\text { Opciones } \\
\text { a y b }\end{array}$ & $\begin{array}{c}\text { Opciones } \\
\text { a y c }\end{array}$ & $\begin{array}{c}\text { Opciones } \\
\text { b y c }\end{array}$ & $\begin{array}{c}\text { Opciones } \\
\text { a, b y c }\end{array}$ & $\begin{array}{c}\text { No } \\
\text { contesta }\end{array}$ \\
\hline $14 \%$ & $80 \%$ & $1.5 \%$ & $0.5 \%$ & $0.5 \%$ & $0 \%$ & $0 \%$ & $3.5 \%$ \\
\hline
\end{tabular}


flora, fauna, recreación y paisaje para las personas; Porque son el lugar donde viven muchas especies de animales'). Para la tercera pregunta, $80 \%$ de los estudiantes respondieron que los parques nacionales son los sitios más importantes para conservar animales y plantas (Tabla 1).
Con respecto a la asignación de las distintas especies de árboles asociadas con bosques nativos de las Yungas Australes (pregunta 4), encontramos que $79 \%$ de los estudiantes identificó a los pinos como pertenecientes a los bosques nativos $\mathrm{y}$, en segundo lugar, al ceibo (Figura 1). En relación con la pregunta

Tabla 2. Porcentaje de estudiantes de séptimo grado en cuatro escuelas primarias de la ciudad de San Salvador de Jujuy que identificaron correcta e incorrectamente (y los nombres que asignaron) las especies de animales nativos de las Yungas Australes ( $n=191$ encuestas realizadas). Además, se da el porcentaje de estudiantes que no contestaron.

Table 2. Percentage of seventh grade students in four elementary schools in the city of San Salvador de Jujuy who correctly and incorrectly identified (and the names they assigned) the species of native animals of the Austral Yungas ( $n=191$ surveys conducted). In addition, is given the percentage of students who did not answer.

\begin{tabular}{|c|c|c|c|c|}
\hline Especie & $\begin{array}{c}\text { Identifica } \\
\text { correctamente }\end{array}$ & $\begin{array}{c}\text { Identifica } \\
\text { incorrectamente }\end{array}$ & $\begin{array}{c}\text { Otros nombres asignados } \\
\text { incorrectamente por los } \\
\text { estudiantes }\end{array}$ & No contesta \\
\hline $\begin{array}{l}\text { 1. Sapo panza roja de las yungas } \\
\text { (Melanophryniscus rubriventris) }\end{array}$ & $17 \%$ & $74 \%$ & $\begin{array}{l}\text { Rana }(63 \%) \\
\text { Rana Venenosa }(6 \%) \\
\text { Rana roja }(1 \%) \\
\text { Otros }(4 \%)\end{array}$ & $9 \%$ \\
\hline $\begin{array}{l}\text { 2. Loro hablador } \\
\text { (Amazona aestiva) }\end{array}$ & $62 \%$ & $29 \%$ & $\begin{array}{l}\text { Guacamayo }(11 \%) \\
\text { Perico }(6 \%) \\
\text { Canario }(4 \%) \\
\text { Otros }(8 \%)\end{array}$ & $9 \%$ \\
\hline $\begin{array}{l}\text { 3. Yaguareté } \\
\text { (Panthera onca) }\end{array}$ & $86 \%$ & $11 \%$ & $\begin{array}{l}\text { Leopardo }(3.5 \%) \\
\text { Puma }(3 \%) \\
\text { Chita }(0.5 \%) \\
\text { Otros }(4 \%)\end{array}$ & $3 \%$ \\
\hline $\begin{array}{l}\text { 4. Tapir } \\
\text { (Tapirus terrestris) }\end{array}$ & $54 \%$ & $33 \%$ & $\begin{array}{l}\text { Oso hormiguero }(23 \%) \\
\text { Chancho de monte }(4 \%) \\
\text { Jabalí }(3 \%) \\
\text { Otros }(3 \%)\end{array}$ & $13 \%$ \\
\hline $\begin{array}{l}\text { 5. Tucán } \\
\text { (Ramphastos toco) }\end{array}$ & $97 \%$ & $0.5 \%$ & Cóndor $(0.5 \%)$ & $2 \%$ \\
\hline $\begin{array}{l}\text { 6. Mirlo de agua } \\
\text { (Cinclus schulzi) }\end{array}$ & $7 \%$ & $63 \%$ & $\begin{array}{l}\text { Hornero }(28 \%) \\
\text { Pájaro }(13 \%) \\
\text { Tero }(9 \%) \\
\text { Otros }(13 \%)\end{array}$ & $30 \%$ \\
\hline $\begin{array}{l}\text { 7. Araña } \\
\text { (Polybetes pythagoricus) }\end{array}$ & $64 \%$ & $29 \%$ & $\begin{array}{l}\text { Tarántula }(24 \%) \\
\text { Araña violinista }(1.5 \%) \\
\text { Araña bananera }(0.5 \%) \\
\text { Otros }(3 \%)\end{array}$ & $7 \%$ \\
\hline $\begin{array}{l}\text { 8. Loro alisero } \\
\text { (Amazona tucumana) }\end{array}$ & $42 \%$ & $40 \%$ & $\begin{array}{l}\text { Guacamayo }(14 \%) \\
\text { Cotorra }(7 \%) \\
\text { Cata }(4 \%) \\
\text { Otros }(15 \%)\end{array}$ & $18 \%$ \\
\hline $\begin{array}{l}\text { 9. Pava de monte alisera } \\
\text { (Penelope dabbenei) }\end{array}$ & $36 \%$ & $39 \%$ & $\begin{array}{l}\text { Pavo real }(27 \%) \\
\text { Pájaro }(5 \%) \\
\text { Águila }(1 \%) \\
\text { Otros }(6 \%)\end{array}$ & $25 \%$ \\
\hline
\end{tabular}




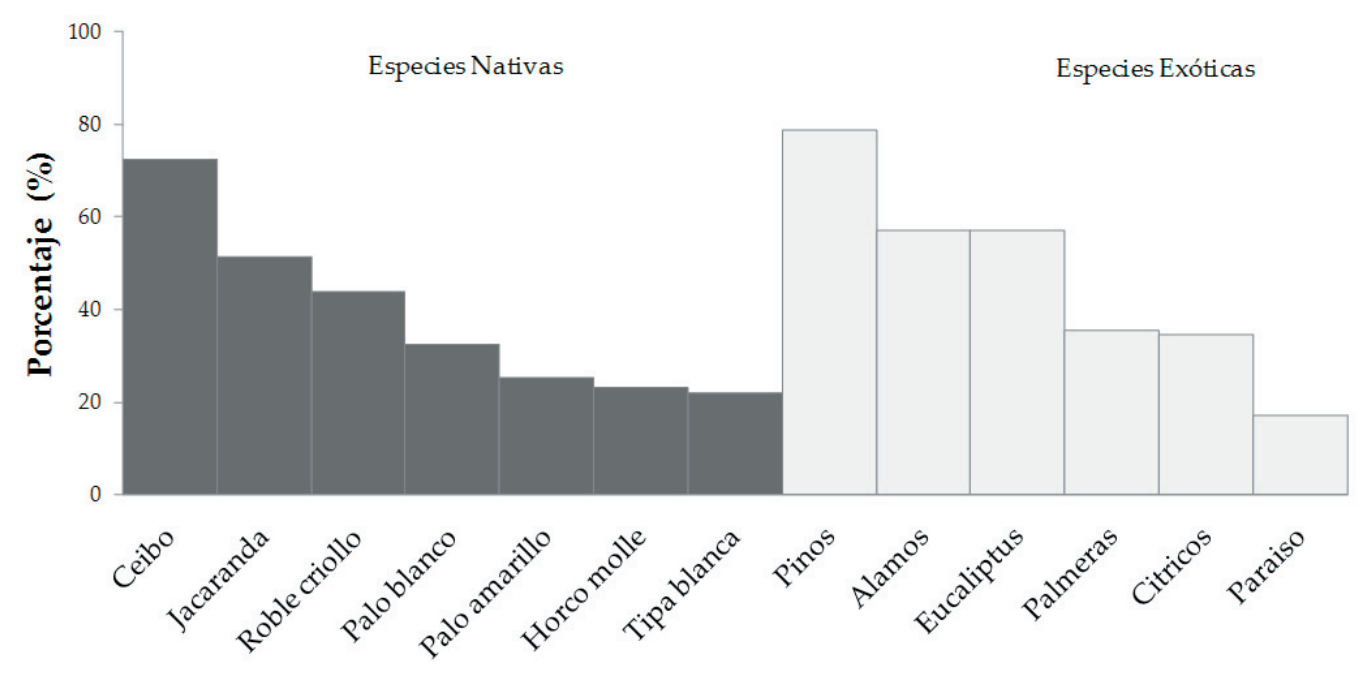

Figura 1. Proporción de estudiantes de séptimo grado de cuatro escuelas primarias de la ciudad de San Salvador de Jujuy que identificaron a las especies de árboles como nativas de las Yungas Australes ( $n=191$ encuestas realizadas). En negro se detallan las especies de árboles nativos de las Yungas Australes y en gris las exóticas.

Figure 1. Proportion of seventh grade students from four elementary schools in the city of San Salvador de Jujuy who identified the tree species as native to the Austral Yungas $(n=191$ surveys conducted). In black the species of native trees of the Austral Yungas and in gray the exotic ones.

5 (especies animales que se pueden encontrar en los bosques nativos de Jujuy), $97 \%$ de los estudiantes identificó correctamente al tucán, el $86 \%$ al yaguareté y el $54 \%$ al tapir. Si bien el loro hablador y la araña de la madera también estuvieron entre las más identificadas (62 y $64 \%$ de las respuestas correctas), los alumnos contestaron sus respuestas con las sinonimias 'loro' y 'arañas' (respuestas que se consideraron correctas) (Tabla 2).

\section{DisCUSIÓN}

Los resultados sugieren que los estudiantes de séptimo grado de las cuatro escuelas encuestadas tienen conocimiento sobre los bosques nativos, los servicios que brindan y la importancia para las especies nativas que lo habitan. Además, identifican a los parques nacionales como una estrategia adecuada para asegurar la conservación de plantas y animales. En parte, esto podría deberse a que las escuelas incorporaron al dictado de las ciencias naturales un enfoque orientado hacia la ecología y el funcionamiento de los ecosistemas naturales, popularizado en los últimos tiempos a partir de material didáctico como videos, láminas y manuales (AdúrizBravo 2001; García 2007). Desde el año 2002, la Secretaría de Medio Ambiente y Desarrollo Sustentable de la Nación impulsa el proyecto 'Bosques Nativos y Áreas Protegidas', y dentro de sus objetivos propone incorporar contenidos vinculados a bosques nativos, biodiversidad y sustentabilidad en las escuelas (SAyDS 2002), lo que también podría contribuir al conocimiento manifestado por los estudiantes.

El mayor desconocimiento detectado en la identificación de especies nativas de árboles y la inclusión de numerosas especies exóticas resulta preocupante (Figura 1). Esto puede deberse a que, en muchos casos, el conocimiento que se adquiere de las plantas es a través de los jardines de las viviendas o de las plazas, donde mayormente se plantan especies exóticas (Campos et al. 2013). Los árboles exóticos pueden transformarse en especies invasoras y causar problemas que afectan a las nativas y al funcionamiento natural de los ecosistemas (Castro-Díez et al. 2004). Por lo tanto, es necesario indagar con los estudiantes en la problemática de las especies exóticas, y que ellos aprendan a reconocer las especies nativas que se encuentran en los bosques y su importancia ecológica para otras especies (Zamora Nasca et al. 2014; Baranzelli et al. 2015). La especie de árbol nativa más identificada como componente de los bosques nativos fue el ceibo. Esto podría estar asociado a que es un símbolo nacional en la Argentina, característica que habría incrementado su popularidad a lo largo de los años, tanto en la sociedad como en las escuelas en particular (Uribarren 2009).

Los animales más reconocidos y correctamente nombrados fueron el tucán y 
el yaguareté, dos especies carismáticas. La ciudad de San Salvador de Jujuy se encuentra rodeada del hábitat natural de los tucanes, por lo que es común avistarlos en la ciudad. Esto facilitaría su reconocimiento por parte de los alumnos, más allá de que es una especie muy llamativa y popular. Por otra parte, la ciudad posee gran cantidad de árboles con frutos que atraen y sirven de alimento a los tucanes. En cuanto al yaguareté, en los últimos años se difundieron ampliamente por los medios de comunicación numerosas campañas de educación acerca de la conservación de esta especie y su hábitat (Di Bitetti et al. 2005; Perovic et al. 2015). También, su reciente implementación como imagen de la moneda nacional favorece a que sea una especie más reconocida. Por otro lado, a pesar de que el mirlo de agua (C. schulzi) y el sapo panza roja de las yungas ( $M$. rubriventris) se pueden encontrar muy cerca de la ciudad, fueron las especies menos identificadas correctamente, probablemente por sus comportamientos y tamaños menos conspicuos. A pesar de que los niños tienen interés y curiosidad por los animales, las escuelas suelen dedicar poco tiempo en estas temáticas, lo que se traduce en escasos conocimientos sobre las especies nativas que los rodean (Barker et al. 2002; Lindemann-Matthies and Bose 2008; Campos et al. 2012, 2013).

Por esta razón, en este estudio queremos enfatizar la importancia de que los estudiantes de escuelas primarias conozcan y valoren la biodiversidad que los rodea. De esta forma se pueden lograr cambios en el conocimiento y la valoración de las especies, ya que es primordial llevar a cabo intervenciones educativas sobre temáticas de biodiversidad y conservación a fin de aumentar el conocimiento (Sudarmadi et al. 2001). Dada la alta tasa de transformación y degradación de las Yungas Australes, es fundamental plantear objetivos de educación en conservación que permitan que las personas tengan un conocimiento sobre los bosques nativos, las especies que los habitan y el valor que poseen para los seres humanos y para el funcionamiento correcto de los ecosistemas (Trombulak et al. 2004; Campos et al. 2013).

Por último, queremos expresar las limitantes de este trabajo y las preguntas que se pueden desarrollar en futuros estudios sobre esta temática. Por un lado, los resultados de este estudio se deben tomar con precaución, ya que la metodología empleada (e.g., la forma en que se plantearon algunas preguntas) puede haber influido sobre las respuestas. Un ejemplo es el uso de sinonimias para identificar las especies, sobre todo en el caso de los loros, que se puede interpretar como una respuesta correcta, pero sin tener conocimiento de qué especie se trata. Por otro lado, trabajos futuros deberán abordar preguntas más específicas, como las planteadas en otros estudios. Por ejemplo, preguntar sobre bosques y biodiversidad a niños que viven en zonas urbanas y rurales, y luego compararlas, como así también separar las respuestas de acuerdo al género (Campos et al. 2013). Esto no fue tratado en este trabajo, ya que el objetivo fue tener una primera apreciación del conocimiento de los niños en los colegios para luego intervenir sobre la base de los resultados registrados. Invertir en manuales, libros, folletos y charlas sobre ecología, bosques y biodiversidad local debe ser una prioridad. Es necesario lograr la conexión entre investigadores en estas temáticas con los responsables de la elaboración de materiales didácticos locales que se puedan difundir en las escuelas. Es indispensable lograr incentivos y establecer mecanismos para que la comunidad científica pueda volcar sus conocimientos al servicio de la sociedad para asegurar la conservación de la biodiversidad.

Agradecimientos. Agradecemos a los estudiantes, maestros y directores de los establecimientos educativos (Escuela $\mathrm{N}^{\circ} 1$ General Manuel Belgrano, Colegio Remedios deEscalada deSan Martín, Complejo Educativo José Hernández y Colegio Nuestra Señora del Huerto) por brindarnos el apoyo necesario para realizar este trabajo. A Juan Repucci por la foto de yaguareté que fue colocada en el poster. Agradecemos a tres revisores anónimos por sus detalles y comentarios que han mejorado en gran medida la calidad del manuscrito. Este trabajo contó con el apoyo y los fondos de la Fundación CEBio (www.cebio.org.ar) y Rufford Small Grant. LR y NP son investigadores de CONICET; AS y SA son becarios doctorales de CONICET. 


\section{REFERENCIAS}

Adúriz-Bravo, A. 2001. La historia de la enseñanza de las ciencias naturales: reflexiones acerca de su emergencia como campo de problemas, a través de una revisión bibliográfica. Revista de Educación 324:365-375.

Baranzelli, M. C., S. A. Córdoba, G. Ferreiro, E. Glinos, C. C. Maubecin, V. Paiaro, and M. E. Renny. 2015. ¿Quién vive ahí?: sobre árboles nativos y exóticos. Una propuesta didáctica para conocer la importancia ecológica del bosque nativo y la problemática de las invasiones biológicas. Revista de Educación en Biología 18:50-64.

Barker, S., D. Slingsby, and S. Tilling. 2002. Ecological fieldwork: Is there a problem. Environmental Education 71: 9-10.

Bickford, D., M. R. C. Posa, L. Qie, A. Campos-Arceiz, and E. P. Kudavidanage. 2012. Science communication for biodiversity conservation. Biol Conserv 151:74-76.

Brown, A. D., S. Pacheco, T. Lomáscolo, and L. Malizia. 2005. Situación ambiental en los bosques andinos yungueños. La situación ambiental argentina. Pp. 53-61.

Brown, A. D., P. Blendinger, T. Lomáscolo, and P. G. Bes. 2009. Selva pedemontana de las Yungas. Historia natural, ecología y manejo de un ecosistema en peligro. San Miguel de Tucumán, Argentina. Ediciones del Subtrópico. Pp. $213-273$.

Campos, C. M., S. Greco, J. J. Ciarlante, M. Balangione, J. B. Bender, J. Nates, and P. Lindemann-Matthies. 2012. Students' familiarity and initial contact with species in the Monte desert (Mendoza, Argentina). Journal of Arid Environments 82:98-105.

Campos, C. M., J. Nates, and P. Lindemann-Matthies. 2013. Percepción y conocimiento de la biodiversidad por estudiantes urbanos y rurales de las tierras áridas del centro-oeste de Argentina. Ecología Austral 23:174-183.

Castro-Díez, P., F. Valladares, and A. Alonso. 2004. La creciente amenaza de las invasiones biológicas. Revista Ecosistemas 13:61-68.

Di Bitetti, M. S., C. De Angelo, A. Paviolo, K. Schiaffino, and P. G. Perovic. 2006. Monumento Natural Nacional en peligro: el desafío de conservar el yaguareté en la Argentina. Pp. 22-34 en Corchera, J. and A. Brown (eds.). La Situación Ambiental Argentina 2005. Fundación Vida Silvestre Argentina. Buenos Aires, Argentina.

Durand, L. 2008. De las percepciones a las perspectivas ambientales: una reflexión teórica sobre la antropología y la temática ambiental. Nueva Antropología 21:75-87.

Furman, M., and M. E. de Podestá. 2010. La aventura de enseñar Ciencias Naturales. Aique Grupo Ed.

García, S. V. 2007. Museos escolares, colecciones y la enseñanza elemental de las ciencias naturales en la Argentina de fines del siglo XIX. História, Ciências, Saúde-Manguinhos 14:173-196.

HuddartొKennedy, E., T. M. Beckley, B. L. McFarlane, and S. Nadeau. 2009. Ruraløurban differences in environmental concern in Canada. Rural Sociology 74: 309-329.

Jordan, R., F. Singer, J. Vaughan, and A. Berkowitz. 2009. What should every citizen know about ecology? Front Ecol Environ 7:495-500.

Lindemann-Matthies, P., and E. Bose. 2008. How many species are there? Public understanding and awareness of biodiversity in Switzerland. Human Ecology 36:731-742.

Perovic, P., S. de Bustos, L. Rivera, S. Arguedas Mora, and L. Lizárraga. 2015. Plan Estratégico para la Conservación del Yaguareté en las Yungas Argentinas. Administración de Parques Nacionales, Secretaría de Ambiente de Salta, Secretaría de Gestión Ambiental de Jujuy y Escuela Latinoamericana de Áreas Protegidas - UCI.

Pilgrim, S. E., L. C. Cullen, D. J. Smith, and J. Pretty. 2008. Ecological Knowledge is Lost in Wealthier Communities and Countries. Environmental Science and Technology 4:1004-1009.

Pooley, J. A., and M. O'Connor. 2000. Environmental education and attitudes: emotions and beliefs are what is needed. Environ Behav 32:711-723.

SAyDS. Secretaría de Ambiente y Desarrollo Sustentable. 2002. Primer Inventario Nacional de Bosques Nativos. Cartografía y Superficie de Bosque Nativo de Argentina. Proyecto Bosques Nativos y Áreas Protegidas. Dirección de Bosques. Secretaría de Ambiente y Desarrollo Sustentable.

Sobel, D. 1999. Beyond ecophobia: Reclaiming the heart in nature education (Nature Literacy Series, Volume 1). Great Barrington, MA, USA. Orion Society.

Sudarmadi, S., S. Suzuki, T. Kawada, H. Netti, S. Soemantri, and A. T. Tugaswati. 2001. A survey of perception, knowledge, awareness, and attitude in regard to environmental problems in a sample of two different social groups in Jakarta, Indonesia. Environment, Development and Sustainability 3:169-183.

Trillo, C., B. Arias Toledo, and S. E. Colantonio. 2016. Uso y percepción del bosque por pobladores de diferente tradición cultural de la Laguna de Mar Chiquita, Córdoba, Argentina. Ecología Austral 26:7-16.

Trombulak, S. C., K.S. Omland, J. A. Robinson, J. J. Lusk, T. L. Fleischner, G. Brown, and M. Domroese. 2004. Principles of conservation biology: Recommended guidelines for conservation literacy from the education committee of the society for conservation biology. Conservation Biology 18:1180-1190.

Uribarren, M. S. 2009. La Comisión Nacional de Museos y de Monumentos y Lugares Históricos de la Argentina entre 1938 y 1946: el patrimonio cultural y la construcción de una idea de nación. Cuadernos de Historia. Serie Economía y Sociedad 11:213-244.

Zamora Nasca, L., L. Montti, R. Grau, and L. Paolini. 2014. Efectos de la invasión del ligustro, Ligustrum lucidum, en la dinámica hídrica de las Yungas del noroeste argentino. Bosque (Valdivia) 35:195-205. 